\title{
Chronic cough is associated with increased reporting of autonomic symptoms
}

\author{
Rachel J. Dockry $\mathbb{B}^{1}$, Carmen L. Farrelly ${ }^{2}$, Joanne Mitchell ${ }^{3}$, Douglas R. Corfield ${ }^{4}$ and Jaclyn A. Smith $\mathbb{B}^{1,3}$
}

${ }^{1}$ Division of Infection, Immunity and Respiratory Medicine, University of Manchester, Manchester, UK. ${ }^{2}$ Division of Musculoskeletal and Dermatological Sciences, University of Manchester, Manchester, UK. ${ }^{3}$ NIHR Manchester Clinical Research Facility at University Hospital of South Manchester, Manchester, UK. ${ }^{4}$ Division of Medical Education, University of Manchester, Manchester, UK.

Corresponding author: Rachel Dockry, Room 120, The Gregson Suite, North West Lung Research Centre, Manchester University Foundation Trust, Wythenshawe Hospital, Southmoor Road, Wythenshawe, Manchester, M23 9LT, UK.

E-mail: rachel.dockry@manchester.ac.uk

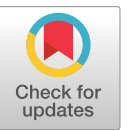

updates

Copyright (C) The authors 2021

This version is distributed under the terms of the Creative Commons Attribution NonCommercial Licence 4.0. For commercial reproduction rights and permissions contact permissions@ersnet.org

Received: 11 Feb 2021 Accepted: 26 April 2021

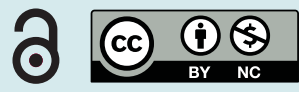

Shareable abstract (@ERSpublications)

Chronic refractory cough patients report a greater range and severity of autonomic symptoms when compared to healthy volunteers. This may suggest that the cough is part of a wider vagal pathology. https://bit.ly/33hzJEt

Cite this article as: Dockry RJ, Farrelly CL, Mitchell J, et al. Chronic cough is associated with increased reporting of autonomic symptoms. ERJ Open Res 2021; 7: 00105-2021 [DOI: 10.1183/23120541.00105-2021].

\section{Abstract}

Background Patients with some neuronal hypersensitivity syndromes experience increased autonomic symptoms. Chronic cough is thought to be a neuronal hypersensitivity disorder and, therefore, may be associated with increased autonomic symptoms.

Methods 96 chronic cough subjects were recruited from the tertiary cough clinic based at Wythenshawe Hospital, Manchester, UK; 76 healthy controls were also recruited. Subjects were aged $>18$ years. Those with significant respiratory disease, significant smoking history or taking medication known to affect cough or autonomic function were excluded. Subjects completed the Composite Autonomic Symptom Score (COMPASS) 31 autonomic symptom questionnaire, the Cough Quality of Life Questionnaire (CQLQ) and a cough severity visual analogue scale (VAS).

Results 96 chronic cough subjects and 76 healthy volunteers were included in the final analysis. MannWhitney U-tests comparing COMPASS 31 scores in both groups showed that the total COMPASS 31 score was significantly higher in the patient group (median 18.4, interquartile range (IQR) 7.5-32.0) than the control group (median 3.6, IQR 1.1-9.5; $\mathrm{p}<0.001$ ). The chronic cough subjects had significantly higher symptom scores than the healthy volunteer groups in all domains $(p \leqslant 0.001)$ except vasomotor symptoms $(\mathrm{p}=0.770)$. There was a positive association between COMPASS 31 and CQLQ in the patient group $(\mathrm{p}<0.001, \mathrm{r}=0.432)$ but not COMPASS 31 and VAS $(\mathrm{p}=0.227)$.

Interpretation Chronic cough patients do indeed report more frequent and severe autonomic symptoms than healthy volunteers, indicating that this population may suffer from dysautonomia. At present, it remains unclear whether this occurs as a result of the cough or whether both the cough and dysfunction are part of some wider vagal pathology.

\section{Introduction}

Chronic coughing is estimated to affect $10 \%$ of the population and can be associated with significant impacts on patients' quality of life. The cough reflex is mediated by the vagus nerve and therefore is intrinsically a component of the autonomic nervous system. Increasing evidence supports the concept that chronic coughing may occur as a consequence of hyperexcitability of the neuronal pathways controlling the cough reflex, which may affect the peripheral and/or central nervous system. For example, patients with chronic cough report coughing in response to innocuous exposures to environmental irritants and activities not normally expected to trigger coughing such as talking/laughing, eating and drinking [1]. It is also well established that they exhibit heightened cough responses to a variety of experimental inhaled irritants [2, 3]. 
However, to date, no study has explored whether chronic cough patients exhibit other symptoms consistent with broader dysregulation of the autonomic nervous system.

Dysautonomia is thought to be associated with a variety of conditions characterised by neuronal/visceral hypersensitivity. Examples include evidence for cardiovascular autonomic impairment in patients with irritable bowel syndrome (IBS) [4], fibromyalgia [5] and complex regional pain syndrome [6]. Chronic cough mainly affects post-menopausal females [7], a demographic which also seems to dominate patient groups suffering from other hypersensitivity disorders such as fibromyalgia [8].

The Composite Autonomic Symptom Score (COMPASS) 31 is a self-assessment instrument designed to capture autonomic symptoms and function. It is an abbreviated version of the Autonomic Symptom Profile, with a simpler scoring system and hence is easier to administer. It has been validated in a range of disorders and shown to have internal consistency [9-11]. It also has clinical value; for example in diabetes, it has a sensitivity of $75 \%$ for detecting the development of cardiac autonomic neuropathy $[11,12]$. It was recently used to track improvements in autonomic symptoms in a phase 3 trial of a novel therapy for transthyretin amyloidosis [13].

The aim of this study was to investigate self-reported autonomic symptoms in chronic cough patients using the COMPASS 31 and compare this to an age- and gender-matched healthy volunteer group, investigating any association with cough severity and associated impacts of cough on quality of life.

Materials and methods

Participants

Chronic cough patients were recruited from the specialist tertiary cough clinic based at Wythenshawe Hospital, Manchester, UK, between 27 June 2014 and 2 March 2017. Healthy volunteers were recruited from hospital staff, responders to advertisements and healthy friends/relatives accompanying patients to their clinic visit. All subjects were over 18 years of age. Chronic cough was defined as cough lasting longer than 8 weeks. Those with significant respiratory disease (e.g., COPD, bronchiectasis, idiopathic pulmonary fibrosis), current smokers or ex-smokers with a significant smoking history (>10 pack-years) and those taking medication known to affect cough or autonomic function were excluded (i.e., neuromodulators such as gabapentin, pregabalin, morphine, amitriptyline). Those with conditions known to be associated with dysautonomia were excluded (i.e., fibromyalgia, diabetes, alcoholism, Parkinson's disease, multiple sclerosis, amyloidosis, postural orthostatic tachycardia syndrome, multiple system atrophy, familial dysautonomia and autoimmune neuropathies) apart from IBS, which is known to be associated with chronic cough. These patients were included with a plan to analyse the effect of this condition on the scores. Healthy volunteers were recruited to the same criteria as the patient group, although a diagnosis of chronic cough was not permitted in this group. This study was approved by Cambridge East Research Ethics Committee (14-EE-0215) and written informed consent was obtained from all participants.

\section{Study design}

Study visits took place either at the NIHR Manchester Clinical Research Facility or in a suitable place arranged with the volunteer. Subject demographics were recorded. Medical history and current medications were ascertained by interviewing the subject. In the chronic cough patient group, duration of cough was recorded. All subjects then completed the questionnaires detailed below.

\section{Questionnaires}

COMPASS 31 is a self-reported, validated, autonomic symptom questionnaire. Covering six domains (orthostatic intolerance, vasomotor, secretomotor, gastrointestinal, bladder and pupillomotor) symptoms are scored 1 for present, 0 for absent and then scored on a scale of frequency, severity and whether they are improving or getting worse. A higher score indicates a greater autonomic symptom burden [9].

The Cough Quality of Life Questionnaire (CQLQ) is a self-reported, validated questionnaire on the impact of cough on daily life. Subjects rate 28 cough-related statements on a four-point Likert scale, ranging from "strongly disagree" to "strongly agree". A higher score, to a maximum of 112, indicates a worse quality of life. Six domains are covered: psychosocial, physical complaints, extreme physical complaints, emotional wellbeing, functional abilities and personal safety fears [14].

For the cough severity visual analogue scale (VAS) subjects place a mark on a 100-mm length line to indicate the current severity of their cough, where $0 \mathrm{~mm}$ is no cough and $100 \mathrm{~mm}$ is the worst cough ever experienced. 


\section{Statistical analysis}

Questionnaire scores were compared between groups using Mann-Whitney U-tests, and subject ages were compared using t-tests (IBM SPSS Statistics V23.0.2; IBM Corp., Armonk, NY, USA). A Spearman's rank correlation was utilised to investigate the relationships between questionnaire scores. Where a subject had failed to answer an item on a questionnaire in error, they were discounted from any analyses involving that questionnaire. A univariate general linear model (GLM) was used to investigate the factors within the study group that influenced the COMPASS 31 total scores and the domain scores.

There are known effects of age and gender on autonomic symptoms, and therefore a smaller dataset composed of paired age- and gender-matched subjects and healthy controls was analysed using the same techniques as the full dataset.

The study sample size of 100 chronic cough patients and 100 healthy volunteers was decided upon to allow adequate power for regression analysis; 10 subjects are required for each parameter to be tested [15].

Results

\section{Participants}

100 chronic cough patients and 76 healthy volunteers completed the study. Owing to errors in questionnaire completion, including missing answers, four chronic cough patients were omitted from the analysis. 96 chronic cough subjects and 76 healthy volunteers were included in the final analysis of questionnaire scores. The demographics of the included subjects are shown in table 1.

A Chi-squared test showed no significant difference between the gender distribution of the groups. The mean age of the chronic cough group (59.7 years) was significantly higher than that of the control group ( 54.70 years), $\mathrm{p}=0.01$, but only by 5 years. In order to ensure that this difference in age was not influencing our findings we repeated all the analyses described below in an age-matched subset of 63 participants. The findings from these analyses were no different from those described below.

\section{Autonomic symptom scores}

The total weighted COMPASS 31 score was significantly higher in the chronic cough group (median 18.4, interquartile range (IQR) 7.5-32.0) than the control group (median 3.6, IQR 1.1-9.5; $\mathrm{p}<0.001$ ) (figure 1). The chronic cough subjects also had significantly higher symptom scores compared with the healthy volunteer group in all domains $(p \leqslant 0.001$,) except for vasomotor symptoms $(p=0.77)$ (figure 2$)$. Removal of the five participants in the chronic cough group with diagnosed IBS did not alter the above findings.

Spearman's rank correlations were performed between the total COMPASS 31 scores, domains and age for both subject groups, and no significant correlations were found. Nonetheless, a smaller dataset comprising

\begin{tabular}{|c|c|c|c|}
\hline & Chronic cough & Healthy volunteers & p-value \\
\hline Subjects $n$ & 96 & 76 & \\
\hline Female $\mathrm{n}(\%)$ & $71(77.1)$ & $54(71.1)$ & 0.732 \\
\hline Age years & $59.7 \pm 12.6$ & $54.7 \pm 12.3$ & $0.01^{\star}$ \\
\hline \multicolumn{4}{|l|}{ Smoking status } \\
\hline Ex-/never-smoker & $22 / 74$ & $15 / 75$ & 0.72 \\
\hline Pack-years & $2.4(1.4-5.8)$ & $3.5(2.2-7.3)$ & 0.26 \\
\hline Cough duration years & $8(5-18)$ & & \\
\hline Cough severity VAS $\mathrm{mm}$ & $50(26-74)$ & & \\
\hline CQLQ score total & $58.3 \pm 13.8$ & & \\
\hline \multicolumn{4}{|l|}{ CQLQ domains } \\
\hline Physical complaints & $18.4 \pm 5.1$ & & \\
\hline Psychosocial & $13.1 \pm 3.7$ & & \\
\hline Functional abilities & $10.2 \pm 3.7$ & & \\
\hline Emotional wellbeing & $5.7 \pm 1.9$ & & \\
\hline Extreme physical complaints & $7.8 \pm 2.3$ & & \\
\hline Personal safety fears & $6.2 \pm 2.4$ & & \\
\hline
\end{tabular}




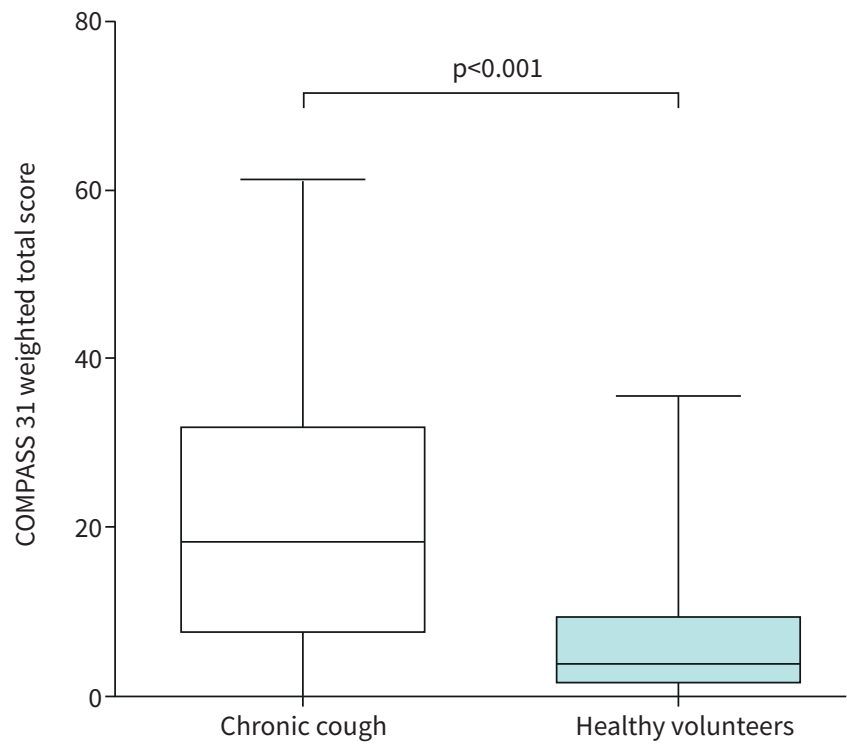

FIGURE 1 Difference in autonomic symptoms scores (Composite Autonomic Symptom Score (COMPASS) 31 weighted scores) between chronic cough patients and healthy volunteers. Data are presented as median, interquartile range and range.

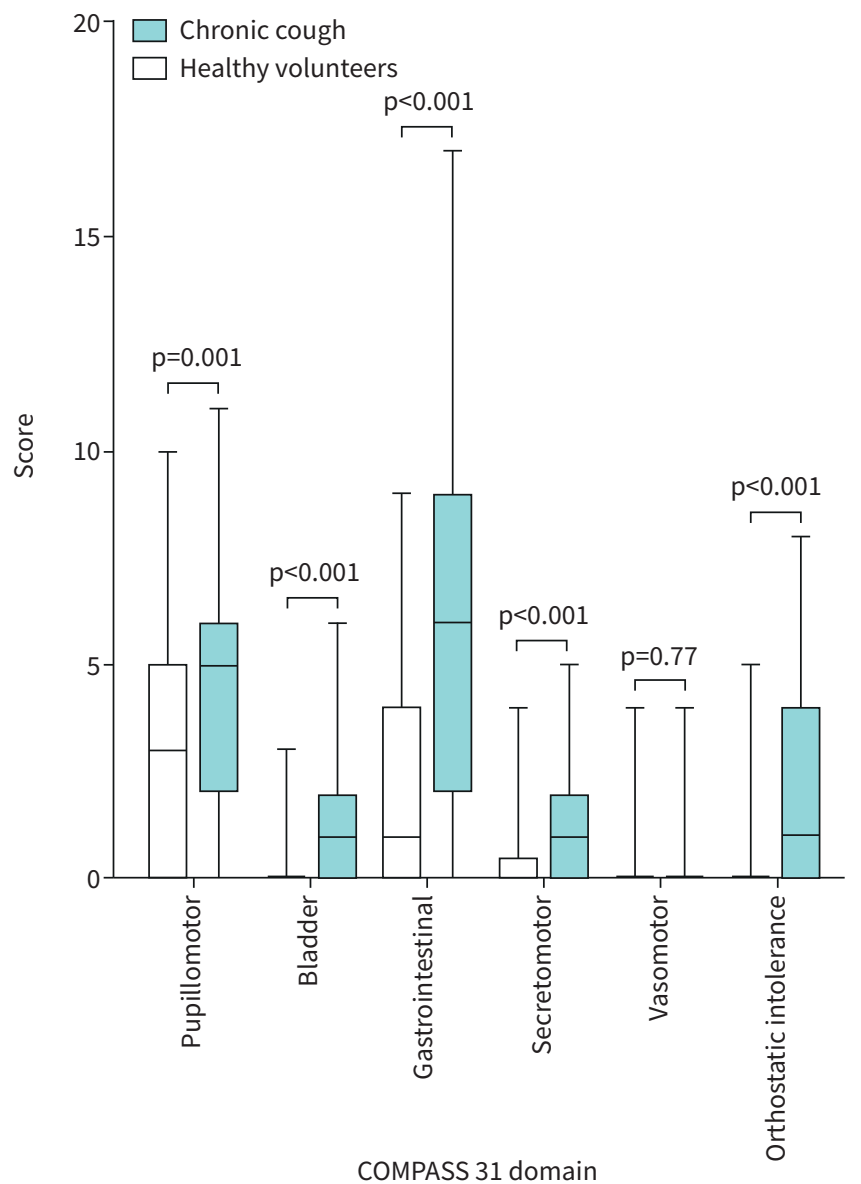

FIGURE 2 Comparison of Composite Autonomic Symptom Score (COMPASS) 31 domain scores for chronic cough patients and healthy volunteers. 
age- and gender-matched chronic cough patients and healthy controls (age matching within 5 years) was also analysed and contained 63 subjects in each group. The difference in age was no longer significant, as expected, with the mean ages of the healthy controls being 54.4 years and chronic cough patients 55.1 years. Mann-Whitney U-tests of the COMPASS 31 weighted total and domain scores repeated the findings of the analysis of the full dataset. Total weighted COMPASS 31 scores were significantly higher in chronic cough patients compared with healthy volunteers (median 16.7 (7.0-29.6) versus 3.6 (1.0-9.4), $\mathrm{p}<0.001$ ). The patients also scored significantly higher in all domains (orthostatic intolerance $\mathrm{p}=0.002$, secretomotor $\mathrm{p}=0.002$, gastrointestinal $\mathrm{p}<0.001$, bladder $\mathrm{p}<0.001$, pupillomotor $\mathrm{p}=0.032$ ) except for the vasomotor symptoms $(\mathrm{p}=0.38)$.

COMPASS 31 score and gender

A comparison of total weighted COMPASS 31 scores between genders within each group was performed using Mann-Whitney U-tests. In the chronic cough group, females (median 21.3, IQR 8.8-33.5) scored significantly higher than males (median 9.6, IQR 4.7-29.0) ( $\mathrm{p}=0.04)$. A similar pattern occurred in the control group, with the females (median 4.7, IQR 1.1-16.0) scoring higher than the males (median 2.6, IQR 0.6-5.5); however, this difference was not significant $(p=0.12)$ (figure 3).

\section{Relationships between cough impact, severity and autonomic symptoms}

The correlation between the chronic cough subjects' CQLQ and COMPASS 31 scores was significant $(\mathrm{p}<0.001, r=0.43)$, with a positive association between the scores (figure 4$)$. In contrast, there was no significant correlation between the cough severity VAS and COMPASS 31 scores $(p=0.23)$.

\section{Regression analysis on COMPASS 31}

GLMs were used to analyse the combined influence of patient's characteristics on the autonomic symptom scores in the chronic cough patients. Complete data for 82 patients were included in the models, as shown in table 2. A model including gender, age, cough duration, CQLQ total score and VAS score explained $24 \%$ of the variation in autonomic symptoms. Only the cough questionnaire scores had a significant influence on the total weighted COMPASS 31 score (CQLQ, $\mathrm{p}<0.001$ and VAS, $\mathrm{p}=0.04$ ).

\section{Discussion}

This is the first study to investigate the prevalence and severity of reported autonomic symptoms in chronic cough. The results suggest that chronic cough patients suffer from a substantial increased autonomic symptom burden when compared to a control group, a difference which persisted when a strictly age- and gender-matched analysis was performed. This increase in symptoms affected a wide range of autonomic systems, with five out of six domains covered by the COMPASS 31 being significantly higher in the patient group. The largest differences were seen in the gastrointestinal and pupillomotor domains, but the

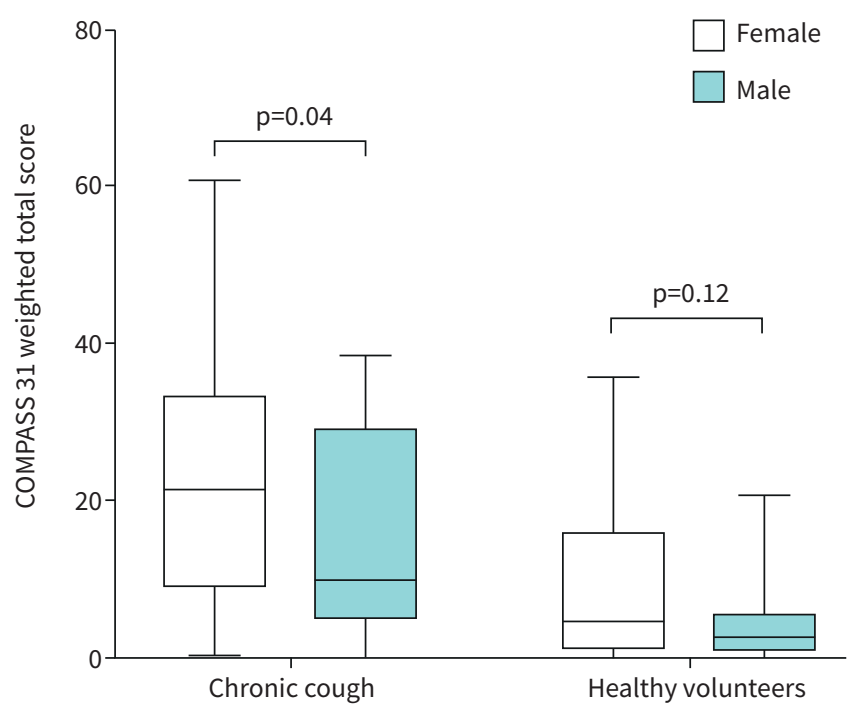

FIGURE 3 Effect of gender on COMPASS 31 total weighted scores in chronic cough patients and healthy volunteers. Data are presented as median, interquartile range and range. 


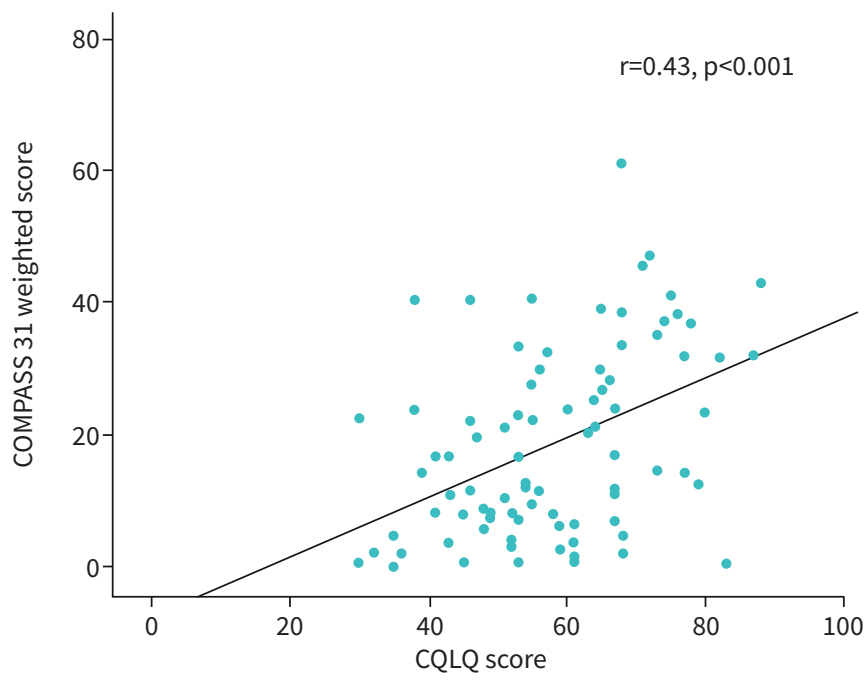

FIGURE 4 Correlation between autonomic symptom scores (Composite Autonomic Symptom Score (COMPASS) 31 weighted scores) and cough-specific quality of life (Cough Quality of Life Questionnaire (CQLQ)).

vasomotor domain score did not differ between groups. Patients with the worst cough-specific quality of life reported the most autonomic symptoms.

The cough reflex is mediated by sensory fibres of the vagus nerve, a fundamental component of the autonomic nervous system. Chronic coughing, that is not productive of sputum, is increasingly thought to occur as a result of hyperexcitability of the neuronal pathways controlling the cough reflex. In many patients this hyperexcitability improves with the treatment of concomitant conditions such as asthma, reflux and nasal disease, but in others either no concomitant conditions are identified or the cough is refractory to treatment of associated conditions (known as refractory chronic cough). Increasing evidence suggests a significant component of the neuronal dysfunction in chronic cough patients resides in the peripheral nervous system [16-18], but there is also evidence for neuronal dysfunction in the central nervous system $[19,20]$. To date, no studies have explored the possibility of broader dysregulation of the autonomic nervous system in patients with chronic cough, although notably chronic cough is reported as a feature of several rare genetic disorders characterised by dysautonomia, i.e. Holmes-Adie syndrome [21], hereditary sensory autonomic neuropathies [22, 23] and cerebellar apraxia neuropathy, vestibular areflexia syndrome $[24,25]$. Our data suggest that chronic cough patients complain of a variety of symptoms suggestive of autonomic imbalance.

The highest COMPASS 31 scores in chronic cough patients were observed in the gastrointestinal and pupillomotor domains. Gastrointestinal items included feeling full after a meal or bloated, vomiting after a meal, having crampy or colicky abdominal pain, diarrhoea, or constipation. Most of these symptoms may occur as consequence of IBS, which has been shown to be associated with chronic cough in one epidemiological study [26], and indeed IBS was diagnosed in five patients in our study. However, even

TABLE 2 Predictors of COMPASS 31 weighted total score, univariate general linear model analysis

p-value

$\begin{array}{lc}\text { Model } & \mathrm{R}^{2}=\mathbf{2 4 . 1} \% \\ \text { Cough duration } & 0.602 \\ \text { Age } & 0.610 \\ \text { CQLQ total score } & <0.001 \\ \text { Cough severity VAS } & 0.040 \\ \text { Gender } & 0.273\end{array}$

COMPASS: Composite Autonomic Symptom Score; VAS: visual analogue scale; CQLQ: Cough Quality of Life Questionnaire. Bold indicates statistical significance. 
when these patients were excluded from the analysis, there was still a significant increase in these gastrointestinal symptoms. The excess of pupillomotor symptoms relates to sensitivity to bright light and focusing problems and cannot be explained by any known associations with chronic cough. It should also be noted that one of the questions on bladder function asks about loss of bladder control. Female patients with chronic cough may experience stress incontinence as a complication of coughing which could account for some of the differences between chronic cough patients and controls in that domain.

The COMPASS 31 scores in this study are comparable to those reported in diabetic cardiac neuropathy, stable multiple sclerosis and patients with systemic sclerosis but lower than in fibromyalgia or patients with postural orthostatic tachycardia syndrome (POTS) [27]. In fibromyalgia, significant correlations have been reported between autonomic symptom burden (measured by COMPASS 31) and measures of heart rate variability indicative of autonomic dysfunction [28]. Similarly, in patients with and without small fibre polyneuropathy COMPASS 31 showed fair diagnostic accuracy versus gold standard autonomic function testing [10].

Chronic cough patients can cough hundreds and even thousands of times a day, and each coughing episode provides a stimulus to the autonomic nervous system resulting in increases in blood pressure and heart rate, especially with bouts of coughing [29]. Over time a desensitisation of the autonomic system, leading to sympathetic dominance and autonomic imbalance similar to that described in obstructive sleep apnoea, could occur [30]. Therefore, it is possible that autonomic dysfunction in patients with chronic cough could occur not only as part of an autonomic neuropathy but also as a consequence of the haemodynamic effects of protracted coughing.

The findings in this study are subject some limitations. Firstly, difficulty in recruiting older eligible healthy volunteers resulted in a slightly younger control group. However, it would seem unlikely that an average age difference of 5 years could account for the differences in autonomic symptoms seen between chronic cough patients and healthy controls. Reassuringly, age was not a significant factor in any of the analyses and the analysis of a strictly age-matched slightly smaller group did not differ from the full dataset. It must also be acknowledged that this observational questionnaire study only captures reported symptoms suggestive of dysautonomia. It is possible that the findings reflect a generalised heightened perception or hypervigilance of symptoms in chronic cough compared with controls, rather than discrete symptoms of autonomic dysfunction. However, counter to this notion, certain domains showed greater differences than others, and the vasomotor symptom domain did not differ at all between chronic cough patients and controls. Both our patient and healthy volunteer groups scored close to zero in this domain, which interestingly is the only completely sympathetically mediated domain, suggesting this could be a result of dysregulation of parasympathetic function and sparing of sympathetic. This autonomic symptom pattern has been reported in other conditions complicated by dysautonomia, e.g. small fibre polyneuropathy [10], Parkinson's disease [31], multiple system atrophy and POTS [27] and autonomic failure secondary to a range of conditions [32]. The lack of change in vasomotor scores in conditions where patients have clinically recognised vasomotor complaints, such as POTS, has caused some authors to speculate that the items in this domain may not adequately capture vasomotor symptoms [27]. Nonetheless, COMPASS 31 has demonstrated increased autonomic symptom burden in a variety of conditions where physiological testing has confirmed dysautonomia [27, 28, 33]. Finally, this was a questionnaire study and therefore collected patient reports of symptoms suggesting dysautonomia. Further investigation with formal autonomic function testing is required to determine whether there is objective evidence of autonomic dysfunction in this patient group.

\section{Conclusion}

This study suggests that chronic cough patients report more frequent and severe autonomic symptoms than healthy volunteers; these symptoms were predominantly seen in parasympathetically mediated systems. Further investigation of these findings including formal autonomic function testing is warranted in this condition.

Acknowledgements: This study was supported by the NIHR Manchester Clinical Research Facility at Wythenshawe Hospital, Manchester NHS Foundation Trust.

Conflict of interest: R.J. Dockry has nothing to disclose. C.L. Farrelly has nothing to disclose. J. Mitchell has nothing to disclose. D.R. Corfield has nothing to disclose. J.A. Smith reports funding for a commercial study to Manchester University NHS Foundation Trust, and personal fees for advisory boards and consultancy work from GlaxoSmithKline; a grant to Christie Hospital, commercial funding for clinical trial and consultancy fees from 
NeRRe Pharmaceuticals; commercial funding for clinical trials and consultancy fees from Menlo and Bayer; consultancy fees from Boehringer Ingelheim, Genentech, Neomed, Chiesi and Bellus; provision of cough monitoring equipment by Vitalograph; a grant to Manchester University NHS Foundation Trust and personal fees from Afferent; funding for a commercial study to Manchester University NHS Foundation Trust and personal fees for consultancy work from Axalbion; and funding for a commercial study to Manchester University NHS Foundation Trust in refractory chronic cough, and personal fees for advisory boards and consultancy work from Merck, all outside the submitted work. In addition, J.A. Smith has a patent (A method for generating output data) licensed.

Support statement: R.J. Dockry and J.A. Smith are funded by a Wellcome Investigator Award, and J.A. Smith is also funded by the NIHR Manchester Biomedical Research Centre and is an NIHR Senior Investigator.

\section{References}

1 Hilton E, Marsden P, Thurston A, et al. Clinical features of the urge-to-cough in patients with chronic cough. Respir Med 2015; 109: 701-707.

2 Belvisi MG, Birrell MA, Khalid S, et al. Neurophenotypes in airway diseases. Insights from translational cough studies. Am J Respir Crit Care Med 2016; 193: 1364-1372.

3 Morice AH, Kitt MM, Ford AP, et al. The effect of gefapixant, a P2X3 antagonist, on cough reflex sensitivity: a randomised placebo-controlled study. Eur Respir J 2019; 54, 1900439.

4 Waring WS, Chui M, Japp A, et al. Autonomic cardiovascular responses are impaired in women with irritable bowel syndrome. J Clin Gastroenterol 2004; 38: 658-663.

5 Kulshreshtha P, Gupta R, Yadav RK, et al. A comprehensive study of autonomic dysfunction in the fibromyalgia patients. Clin Auton Res 2011; 22: 117-122.

6 Terkelsen AJ, Mølgaard H, Hansen J, et al. Heart rate variability in complex regional pain syndrome during rest and mental and orthostatic stress. Anesthesiology 2012; 116: 133-146.

7 Kavalcikova-Bogdanova N, Buday T, Plevkova J, et al. Chronic cough as a female gender issue. Adv Exp Med Biol 2016; 905: 69-78.

8 Jones GT, Atzeni F, Beasley M, et al. The prevalence of fibromyalgia in the general population: a comparison of the American College of Rheumatology 1990, 2010, and modified 2010 classification criteria. Arthritis Rheumatol 2015; 67: 568-575.

9 Sletten DM, Suarez GA, Low PA, et al. COMPASS 31: a refined and abbreviated Composite Autonomic Symptom Score. Mayo Clin Proc 2012; 87: 1196-1201.

10 Treister R, O'Neil K, Downs HM, et al., Validation of the composite autonomic symptom scale 31 (COMPASS-31) in patients with and without small fiber polyneuropathy. Eur J Neurol 2015; 22: 1124-1130.

11 Greco C, Di Gennaro F, D'Amato C, et al. Validation of the Composite Autonomic Symptom Score 31 (COMPASS 31) for the assessment of symptoms of autonomic neuropathy in people with diabetes. Diabet Med 2017; 34: 834-838.

12 D'Amato C, Greco C, Lombardo G, et al. The diagnostic usefulness of the combined COMPASS 31 questionnaire and electrochemical skin conductance for diabetic cardiovascular autonomic neuropathy and diabetic polyneuropathy. J Peripher Nerv Syst 2020; 25: 44-53.

13 Gonzalez-Duarte A, Berk JL, Quan D, et al. Analysis of autonomic outcomes in APOLLO, a phase III trial of the RNAi therapeutic patisiran in patients with hereditary transthyretin-mediated amyloidosis. J Neurol 2020; 267: 703-712.

14 French CT, Irwin RS, Fletcher, KE, et al. Evaluation of a cough-specific quality-of-life questionnaire. Chest 2002; 121: 1123-1131.

15 Peduzzi P, Concato J, Kemper E, et al. A simulation study of the number of events per variable in logistic regression analysis. J Clin Epidemiol 1996; 49: 1373-1379.

16 Shapiro CO, Proskocil BJ, Oppegard LJ, et al. Airway sensory nerve density is increased in chronic cough. Am J Respir Crit Care Med 2020; 203: 348-355.

17 Abdulqawi R, Dockry R, Holt K, et al. P2X3 receptor antagonist (AF-219) in refractory chronic cough: a randomised, double-blind, placebo-controlled phase 2 study. Lancet 2015; 385: 1198-1205.

18 Smith JA, Kitt MM, Butera P, et al. Gefapixant in two randomised dose-escalation studies in chronic cough. Eur Respir J 2020; 55: 1901615.

19 Hilton E, Satia I, Holt K, et al. The effect of pain conditioning on experimentally evoked cough: evidence of impaired endogenous inhibitory control mechanisms in refractory chronic cough. Eur Respir J 2020; 56: 2001387.

20 Ando A, Smallwood D, McMahon M, et al. Neural correlates of cough hypersensitivity in humans: evidence for central sensitisation and dysfunctional inhibitory control. Thorax 2016; 71: 323-329.

21 Kimber J, Mitchell D, Mathias CJ. Chronic cough in the Holmes-Adie syndrome: association in five cases with autonomic dysfunction. J Neurol Neurosurg Psychiatry 1998; 65: 583-586. 
22 Spring PJ, Kok C, Nicholson GA, et al. Autosomal dominant hereditary sensory neuropathy with chronic cough and gastro-oesophageal reflux: clinical features in two families linked to chromosome 3p22-p24. Brain 2005; 128: 2797-2810.

23 Miura S, Shibata $\mathrm{H}$, Kida $\mathrm{H}$, et al. Hereditary motor and sensory neuropathy with proximal dominancy in the lower extremities, urinary disturbance, and paroxysmal dry cough. J Neurol Sci 2008; 273: 88-92.

24 Infante J, Garcia A, Serrano-Cardenas KM, et al. Cerebellar ataxia, neuropathy, vestibular areflexia syndrome (CANVAS) with chronic cough and preserved muscle stretch reflexes: evidence for selective sparing of afferent la fibres. J Neurol 2018; 265: 1454-1462.

25 Szmulewicz DJ, McLean CA, MacDougall HG, et al. CANVAS an update: clinical presentation, investigation and management. J Vestib Res 2014; 24: 465-474.

26 Ford AC, Forman D, Moayyedi P, et al. Cough in the community: a cross sectional survey and the relationship to gastrointestinal symptoms. Thorax 2006; 61: 975-979.

27 Rea NA, Campbell CL, Cortez MM. Quantitative assessment of autonomic symptom burden in Postural tachycardia syndrome (POTS). J Neurol Sci 2017; 377: 35-41.

28 Kang JH, Kim JK, Hong SH, et al. Heart rate variability for quantification of autonomic dysfunction in fibromyalgia. Ann Rehabil Med 2016; 40: 301-309.

29 Wei JY, Harris WS. Heart rate response to cough. J Appl Physiol Respir Environ Exerc Physiol 1982; 53: 1039-1043.

30 Abboud F, Kumar R. Obstructive sleep apnea and insight into mechanisms of sympathetic overactivity. J Clin Invest 2014; 124: 1454-1457.

31 Kim Y, Seok JM, Park J, et al. The composite autonomic symptom scale 31 is a useful screening tool for patients with Parkinsonism. PLoS One 2017; 12: e0180744.

32 Pierangeli G, Turrini A, Giannini G, et al. Translation and linguistic validation of the Composite Autonomic Symptom Score COMPASS 31. Neurol Sci 2015; 36: 1897-1902.

33 Cambras T, Castro-Marrero J, Zaragoza MC, et al. Circadian rhythm abnormalities and autonomic dysfunction in patients with Chronic Fatigue Syndrome/Myalgic Encephalomyelitis. PLOS ONE 2018; 13: e0198106. 